\title{
Recurrent Ischemic Stroke Treated With Mechanical Thrombectomy During a Pregnancy: a case report
}

\author{
Jakubicek $\mathrm{S}^{1}$, Vanicek $\mathrm{J}^{2}$, Pavelek $\mathrm{Z}^{3}$, Reif $\mathrm{M}^{1}$ \\ ${ }^{1}$ Department of Neurology, St. Anne's University Hospital Brno, Czech Republic \\ 2 Department of Radiology, St. Anne's University Hospital Brno, Czech Republic \\ ${ }^{3}$ Department of Neurology, University Hospital Hradec Kralove, Czech Republic
}

\section{INTRODUCTION}

Although the evidence from large randomized controlled trials confirmed that mechanical thrombectomy is effective in selected patients with an intracranial proximal artery occlusion, pregnancy was excluded from the study protocols. The described case depicts an endovascular treatment of recurrent acute ischemic stroke in pregnancy

\section{CASE REPORT}

A 19-year-old woman during her 12th gestational week was admitted to our comprehensive stroke center suffering from acute ischemic stroke within the territory of left internal carotid artery. The National Institute of Health Stroke Scale (NIHSS) during admission was 17. She had a history of aneurysmal subarachnoid hemorrhage, endocarditis 4 years ago and ischemic stroke with occlusion of M2 segment of left middle cerebral artery (MCA) successfully treated with the mechanical thrombectomy (at discharge NIHSS 0) seventeen days before recent stroke.

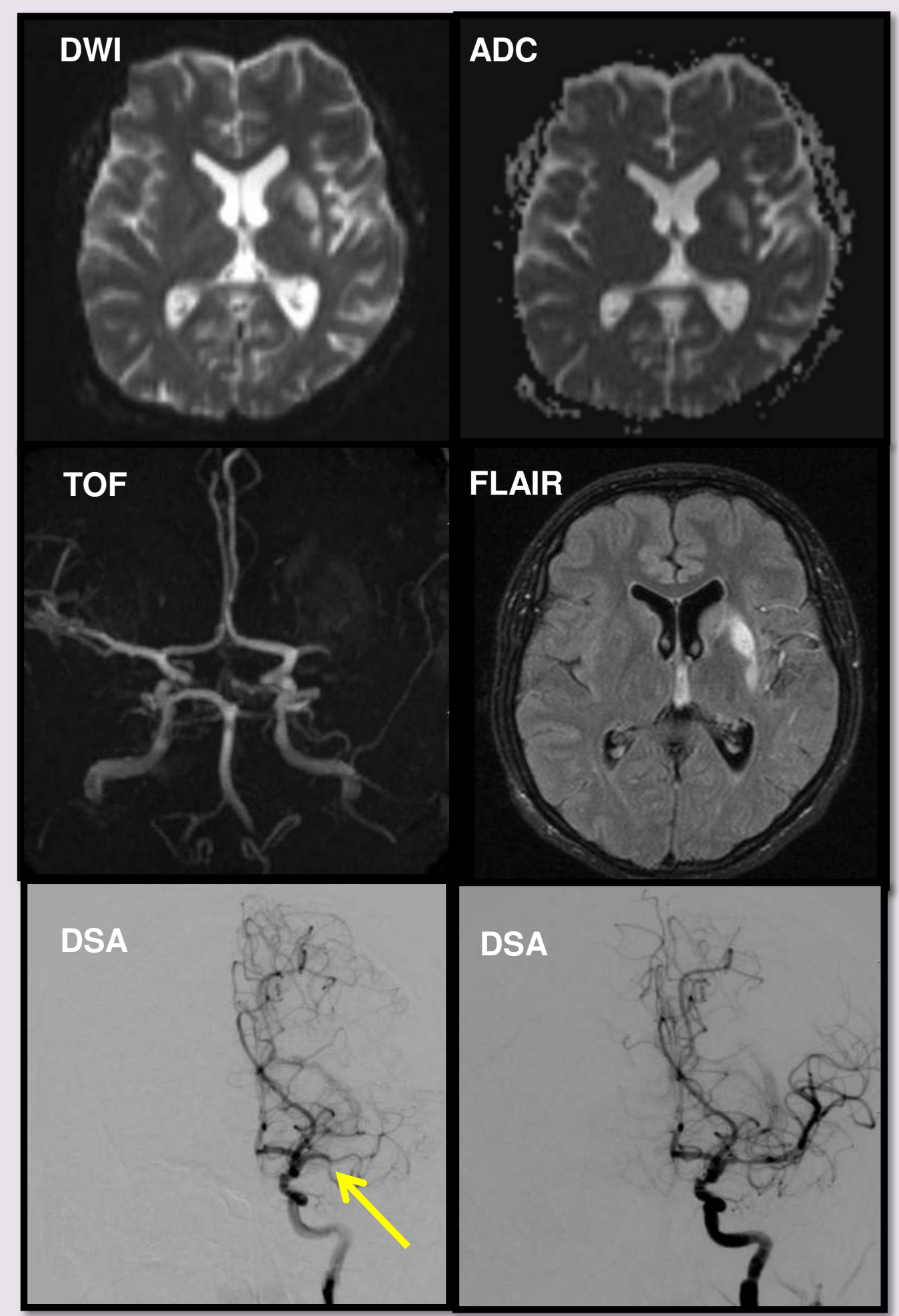

Figure 1. MRI at admission, DSA with left MCA occlusion before (yellow arrow) and after mechanical thrombectomy

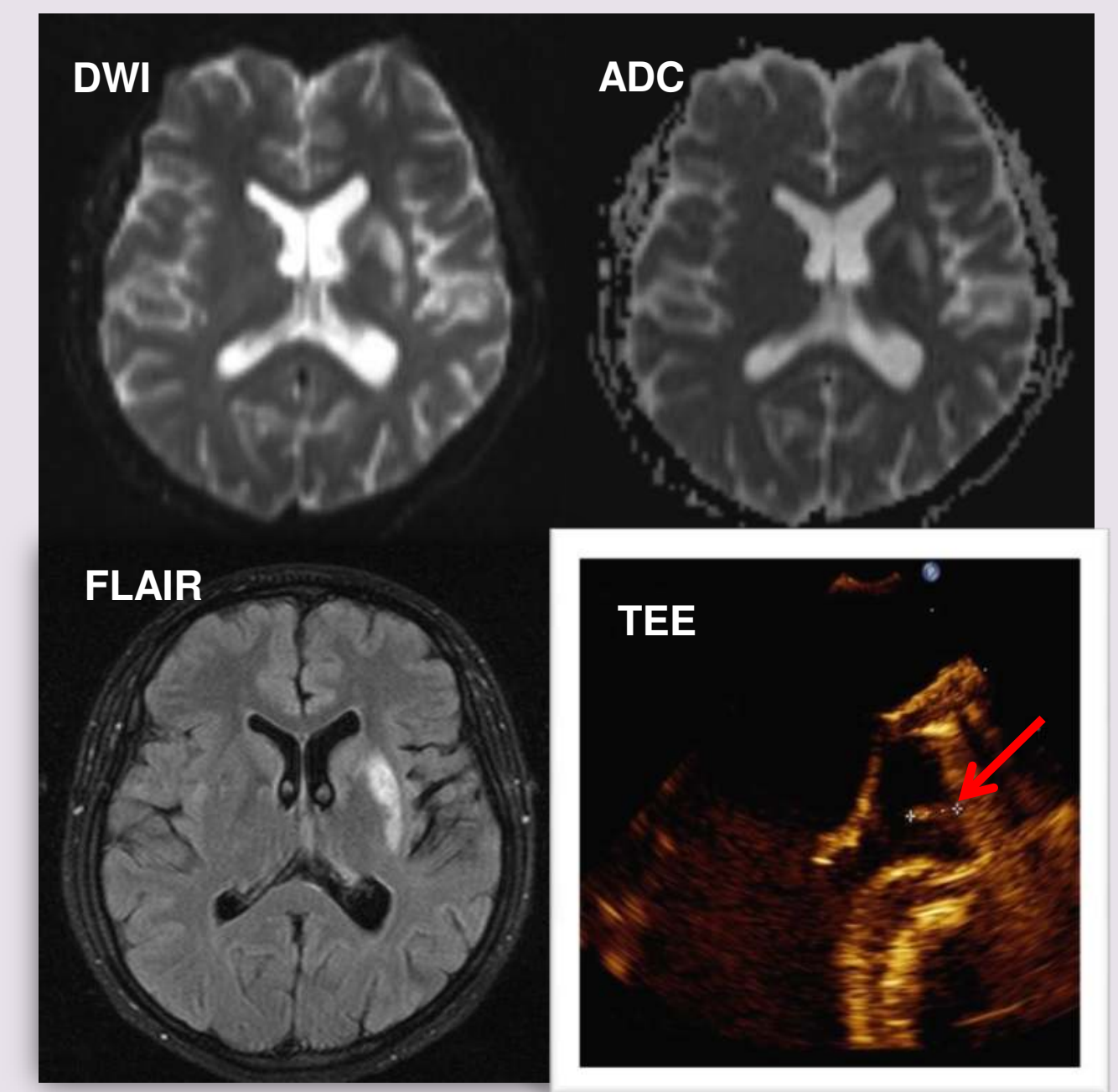

Figure 2. MRI after 24 hours. TEE with thrombus in left ventricular outflow tract (red arrow).
The urgent MRI at our hospital showed an acute and subacute ischemic lesion involving the left basal ganglia and the occlusion of M1 segment of left MCA (Figure 1). Due to her above mentioned history, she was treated with mechanical thrombectomy alone using stent retriever. Complete recanalization was achieved with no evidence of hemorrhagic transformation on the control MRI. Transesophageal echocardiography identified thrombus in left ventricular outflow tract (Figure 2). The patient was discharged with NIHSS 0 and received anticoagulation therapy.

\section{CONCLUSION}

Treatment of stroke with even repeated mechanical thrombectomy within 17 days apart was in this case of pregnant woman feasible and successful and might be considered as option for treatment of sever ischemic stroke with M1 or M2 occlusion in pregnancy. 